Ergod. Th. \& Dynam. Sys. (1987), 7, 119-132

Printed in Great Britain

\title{
On Gibbs and equilibrium states
}

\author{
NICOLAI T. A. HAYDN \\ Mathematics Institute, University of Warwick, Coventry, CV4 7AL, England
}

(Received 25 July 1985 and revised 21 February 1986)

Abstract. Ruelle and Capocaccia gave a new definition of Gibbs states on Smale spaces. Equilibrium states of suitable functions thereon are known to be Gibbs states. The converse is discussed in this paper, where the problem is reduced to shift spaces and solved there by constructing suitable conjugating homeomorphisms in order to verify the conditions for Gibbs states which Bowen gave for shift spaces, where the equivalence to equilibrium states is known.

Let $(\Omega, T)$ be a Smale space, then for any continuous function $F: \Omega \rightarrow \mathbb{R}$ the pressure $P(T, F)$ can be defined by the variational principle

$$
P(T, F)=\sup _{\rho}\left(h_{T}(\rho)+\int_{\Omega} F d \rho\right),
$$

where $\rho$ runs over all $T$-invariant probability measures over $\Omega$. Here $h_{T}(\rho)$ is the measure theoretic entropy with respect to $T$ and $\rho$. A measure for which the supremum is attained is called an equilibrium state. For every Hölder continuous $F$ there is a unique equilibrium state if $(\Omega, T)$ is topologically mixing (cf. [ 1 , Theorem 1.22]).

Let $d(\cdot, \cdot)=d_{\Omega}(\cdot, \cdot)$ denote a metric on $\Omega$. A map $\psi$ from some open $U \subset \Omega$ into $\Omega$ is called conjugating if $d\left(T^{k} \circ \psi(x), T^{k}(x)\right) \rightarrow 0$ for $|k| \rightarrow \infty$ uniformly in $x \in U$. Actually, as one may derive from lemma 4, the distance decreases in a uniformly exponential way with a properly chosen metric.

Definition 1. (cf. [2] and [4].) Let $F$ be a Hölder continuous real valued function on $\Omega$. A probability measure $\nu$ is called a Gibbs state for $F$ if

where

$$
\int_{U}(\tau \circ \psi) g d \nu=\int_{\psi(U)} \tau d \nu
$$

$$
g(z)=\exp \sum_{k \in \mathbf{Z}}\left(F \circ T^{k} \circ \psi(z)-F \circ T^{k}(z)\right),
$$

for all bounded and measurable functions $\tau: \psi(U) \rightarrow \mathbb{R}$ and all conjugating homeomorphisms $\psi: U \rightarrow \psi(U)$, where $U=U_{\psi}$ is an open set in $\Omega$.

Equilibrium states for $F$ are also Gibbs states for $F$. This is proved in Ruelle's book [4, theorem 7.18]. The converse, there referred to as an open question (cf. [1, p. 170]), will be demonstrated in this paper, i.e.

THEOREM 2. Let $(\Omega, T)$ be a Smale space and $F \in C^{\theta}(\Omega)$, for $\theta \in(0,1)$, i.e., $F$ is a Hölder continuous real valued function over $\Omega$ with exponent $\theta$, and let $\nu$ be a Gibbs 
state for $F$. Then there is a number $t \in \mathbb{N}$ such that $\nu$ is invariant under $T^{t}$ and is an equilibrium state for $F_{t}=\sum_{0 \leq r<t} F \circ T^{r}$ on $\left(\Omega, T^{t}\right)$.

The proof is by a sequence of lemmas. We begin by introducing a Markov partition on the Smale space $(\Omega, T)$, which gives rise to a shift space $\left(\Sigma_{A}, \sigma\right)$. Most of the proof will be treated on this symbolic level. In order to see that every Gibbs measure on $(\Omega, T)$ lifts in a well-defined way to one on $\left(\Sigma_{A}, \sigma\right)$ it is shown that the boundary set of any Markov partition has measure zero. This is done first in lemma 5 for $T$-invariant Gibbs states and then generalised in proposition 6 for non-invariant Gibbs measures. For constructing conjugating homeomorphisms on $\left(\Sigma_{A}, \sigma\right)$ an obvious method exists, which will be used at three stages in this paper: first in lemma 7 to estimate the measure of cylinder sets; secondly in lemma 9 to show that the wandering set has measure zero, and finally in lemma 10 to prove that Gibbs states on shift spaces $\left(\Sigma_{A}, \sigma\right)$ with $\sigma$ acting topologically mixing, are invariant under $\sigma$. This all taken together (proposition 11 ), provided $\sigma$ is topologically mixing, verifies the definition Bowen gave for Gibbs states (cf. [1, Theorem 1.2]), for which it is known that they are equilibrium states on $\left(\Sigma_{A}, \sigma\right)$. By standard results the same holds true for $(\Omega, T)$. To justify the construction of conjugating homeomorphisms on $\left(\Sigma_{A}, \sigma\right)$ we begin in lemma 4 by proving that they can be 'pushed down' to conjugating homeomorphisms on $(\Omega, T)$.

From now on $\nu$ denotes a Gibbs state for $F$ on $(\Omega, T)$.

Let $x$ be any point in $\Omega$; then there exist a positive number $\delta$ and a number $\lambda \in(0,1)$, such that:

$$
d\left(T^{j}(y), T^{j}(z)\right) \leq l \cdot \lambda^{|j|} d(y, z) \text { if } \begin{cases}y, z \in V_{x}^{+}(\delta) & \text { for } j \geq 0, \\ y, z \in V_{x}^{-}(\delta) & \text { for } j<0,\end{cases}
$$

for a positive constant $l$ and where $V_{x}^{+}(\delta)\left(V_{x}^{-}(\delta)\right)$ denotes the stable (unstable) manifold through $x$ cut off at distance $\delta$. The constant $l$ can be assumed to be 1 , $d(\cdot, \cdot)$ is then an adapted metric, which always exists. Furthermore $T$ is expansive, i.e. there exists a positive constant $\varepsilon$ such that for two points $x, x^{\prime} \in \Omega$, with $x \neq x^{\prime}$ there is an index $n \in \mathbb{Z}$ for which $d\left(T^{n}(x), T^{n}\left(x^{\prime}\right)\right)>\varepsilon$. There exist Markov partitions with arbitrarily small diameter (cf. [1]). We choose one, $\{R[j]: j \in \mathscr{A}\}$ over an alphabet $\mathscr{A}$, such that $\operatorname{diam} R[j]<\varepsilon / 2$ for all $j \in \mathscr{A}$. The sets $R[j]$ are called rectangles. Let $A$ be the corresponding transition matrix and define the shift space

$$
\Sigma_{A}=\left\{z: z=\left\{z_{i}: i \in \mathbb{Z}\right\}, \quad A\left[z_{i}, z_{i+1}\right]=1 \quad \text { for all } i \in \mathbb{Z}\right\},
$$

which, endowed with the metric $d_{\Sigma}(x, y)=\lambda^{k}$, where $k=\max \left\{j: x_{i}=y_{i}\right.$ for all $\left.|i|<j\right\}$, is a metric space. The surjection $\pi: \Sigma_{A} \rightarrow \Omega$ is Lipshitz continuous with constant, say, $L$. See also $\left[4, p\right.$. 130]. The two-sided shift $\sigma$ on $\Sigma_{A}$ is defined by $\sigma(z)=z^{\prime}$ where $z_{i}^{\prime}=z_{i+1}$ for all $i \in \mathbb{Z}$, and covers $T$ on $\Omega: T \circ \pi=\pi \circ \sigma$. We will need:

LEMMA 3. Let $\varepsilon_{0}, \varepsilon_{1}$ be expansive constants, then

(i) Given $\delta>0$ and let $M_{\delta}$ be the maximal number, such that $y, y^{\prime} \in \Omega$ with $d_{\Omega}\left(y, y^{\prime}\right)<\delta$ implies $d_{\Omega}\left(T^{j}(y), T^{j}\left(y^{\prime}\right)\right)<\varepsilon_{0}$ for all $|j|<M_{\delta} ;$ then $M_{\delta} \rightarrow \infty$ as $\delta$ approaches zero. 
(ii) Given $M \in \mathbb{N}$ and let $\delta_{M}^{\prime}$ be the smallest number such that $y, y^{\prime} \in \Omega$ with $d_{\Omega}\left(T^{j}(y)\right.$, $\left.T^{j}\left(y^{\prime}\right)\right)<\varepsilon_{1}$ for all $|j|<M$ implies $d_{\Omega}\left(y, y^{\prime}\right)<\delta_{M}^{\prime}$; then $\delta_{M}^{\prime} \rightarrow 0$ as $M \rightarrow \infty$.

The proof is easy; see e.g. [1].

LEMMA 4. Let $\varphi: U \rightarrow \varphi(U)$, for $U \subset \Sigma_{A}$ be a uniformly continuous conjugating homeomorphism for $\sigma$, then there is a finite decomposition on $U=\bigcup_{i} U_{i}$ and conjugating homeomorphisms $\psi_{i}: V_{i}=\pi\left(U_{i}\right) \rightarrow \psi_{i}\left(V_{i}\right), V_{i} \subset \Omega$, for $T$, which are projections of $\varphi$ on $U_{i}$, i.e. $\psi_{i} \circ \pi=\pi \circ \varphi$ on $U_{i}$ for all $i$.

Proof. There is an $n^{*} \in \mathbb{N}$, such that $(\varphi(z))_{i}=z_{i}$ for all $|i| \geq n^{*}$ and $z \in U$. Otherwise there would be a sequence $\left\{z^{k}: k \in \mathbb{N}\right\}$, such that $\left(\varphi\left(z^{k}\right)\right)_{i[k]} \neq z_{i[k]}^{k}$ for a sequence $|i[k]| \rightarrow \infty$ as $k \rightarrow \infty$. Or $d_{\Sigma}\left(\sigma^{i[k]} \circ \varphi\left(z^{k}\right), \sigma^{i[k]}\left(z^{k}\right)\right)=1$ for all $k \in \mathbb{N}$, but this contradicts the fact that $d_{\Sigma}\left(\sigma^{j} \circ \varphi(z), \sigma^{j}(z)\right)$ converges to zero uniformly in $z$.

By uniform continuity there exists a $\delta \in(0,1)$, such that $d_{\Sigma}(x, y) \leq \delta$ implies $d_{\Sigma}(\varphi(x), \varphi(y)) \leq \lambda^{n^{*}}$. Now set

$$
n=\max \left(n^{*},[\log \delta / \log \lambda+1]\right) .
$$

If for two points $x, y \in U$ we have $x_{i}=y_{i}$ for $|i|<n$ the same is true for their images under $\varphi:(\varphi(x))_{i}=(\varphi(y))_{i}$ for all $|i|<n$. Select a finite number of points $\left\{y^{i}\right.$ : $i \in \mathscr{I}\} \subset U$, with $|\mathscr{I}|<\infty$, and set

$$
U_{i}=\left\{x: x_{j}=y_{j}^{i} \quad \forall|j|<n\right\} \cap U .
$$

A good choice of this set yields $U=\bigcup_{i \in \mathscr{I}} U_{i}$.

The rectangles $R[i], R[j]$ are said to be related if $R[i] \cap R[j] \neq \varnothing$. Write $\partial^{+} R[j]$ and $\partial^{-} R[j]$ for the forward and backward oriented parts of the boundary of $R[j]$, and set $\partial R=\bigcup_{j \in \mathscr{A}}\left(\partial^{+} R[j] \cup \partial^{-} R[j]\right)$. Call the entire boundary set $K=$ $\bigcup_{k \in \mathbf{Z}} T^{k}(\partial R)$.

The components of two points in $\pi^{-1}(K) \subset \Sigma_{A}$ always denote pairs of related rectangles. The same is true for their images under $\varphi$ if we restrict to $U_{i}$ for all $i \in I$. To see this, choose any two points $x, x^{\prime} \in U_{i}$ for which $\pi(x)=\pi\left(x^{\prime}\right)$, then $R\left[x_{j}\right]$ and $R\left[x_{j}^{\prime}\right]$ are related for all $j \in \mathbb{Z}$. By construction of $U_{i}$ their images have the same components for $|j|<n$ and so $R\left[(\varphi(x))_{j}\right]$ and $R\left[\left(\varphi\left(x^{\prime}\right)\right)_{j}\right]$ are again related for all $j \in \mathbb{Z}$. Since

$$
d_{\Omega}\left(T^{j} \circ \pi \circ \varphi(x), T^{j} \circ \pi \circ \varphi\left(x^{\prime}\right)\right) \leq \operatorname{diam}\left(R\left[(\varphi(x))_{j}\right] \cup R\left[\left(\varphi\left(x^{\prime}\right)\right)_{j}\right]\right)<\varepsilon,
$$

for all $j \in \mathbb{Z}$, we conclude that $\pi \circ \varphi(x)=\pi \circ \varphi\left(x^{\prime}\right)$, since $\varepsilon$ is an expansive constant (cf. [3]).

Define on $V_{i}=\pi\left(U_{i}\right), \psi_{i}: x \rightarrow \pi \circ \varphi\left(\pi^{-1}(x) \cap U_{i}\right)$. The sets $V_{i}$ are not necessarily open, but contain open subsets. The maps $\psi_{i}$ will turn out to be conjugating and injective maps on $V_{i}$. Set

$$
\beta=[1+|\log (\varepsilon /(4 L)) / \log \lambda|]
$$

and take for each $i$ a finite cover $\left\{U_{i, k}: k \in \mathscr{K}_{i}\right\}$ of $U_{i}$ with $\left|\mathscr{K}_{i}\right|<\infty$, where $U_{i, k}=$ $\left\{x: x_{j}=z_{j}^{k}, \forall|j|<n+\beta\right\}$ for finite subsets $\left\{z^{k}: k \in \mathscr{K}_{i}\right\}$ chosen as before. It remains to be shown that the maps $\psi_{i}$ are continuous. This is done for each $U_{i, k}$, the region being extended to the closures of the $\pi\left(U_{i, k}\right)$ in $\pi\left(U_{i}\right)$. 
Choose any two points $x, x^{\prime} \in U_{i, k}$ with $y=\pi(x)$ and $y^{\prime}=\pi\left(x^{\prime}\right)$, i.e. $y, y^{\prime} \in V_{i, k}=$ (cl $\left.\pi\left(U_{i, k}\right)\right) \cap \pi\left(U_{i}\right)$. Now we apply lemma 3 (i) setting $\varepsilon_{0}=\varepsilon / 2$ (and restrict to $\delta$, so that $\left.M_{\delta}>n+\beta\right)$. From the construction of the $V_{i, k}$ it is clear that $T^{j} \circ \pi \circ \varphi(x)$ and $T^{j} \circ \pi \circ \varphi\left(x^{\prime}\right)$ travel under $T$ through the same rectangles for $|j|<n+\beta$. Estimate

$$
\begin{aligned}
d_{\Omega}\left(T^{j} \circ \psi_{i} \circ \pi(x), T^{j} \circ \psi_{j} \circ \pi\left(x^{\prime}\right)\right) \\
\quad \leq L\left\{d_{\Sigma}\left(\sigma^{j} \circ \varphi(x), \sigma^{j}(x)\right)+d_{\Sigma}\left(\sigma^{j}\left(x^{\prime}\right), \sigma^{j} \circ \varphi\left(x^{\prime}\right)\right)\right\}+d_{\Omega}\left(T^{j} \circ \pi(x), T^{j} \circ \pi\left(x^{\prime}\right)\right) \\
\quad \leq 2 L \lambda^{|j|-n}+\varepsilon / 2 \leq \varepsilon,
\end{aligned}
$$

if $n+\beta \leq|j|<M_{\delta}$. Hence $d_{\Omega}\left(T^{j} \circ \psi_{i}(y), T^{j} \circ \psi_{i}\left(y^{\prime}\right)\right) \leq \varepsilon$ for all $|j|<M_{\delta}$ and we conclude using lemma 3 (ii) with $\varepsilon_{1}=\varepsilon$, that $d_{\Omega}\left(\psi_{i}(y), \psi_{i}\left(y^{\prime}\right)\right) \leq \delta^{\prime}$ where $\delta^{\prime} \rightarrow 0$ as $\delta$ approaches zero. Moreover, $\psi_{i}$ is a continuous homeomorphism onto the boundaries $\partial\left(\pi\left(U_{i, k}\right)\right) \cap \partial\left(\pi\left(U_{i, l}\right)\right) \cap \pi\left(U_{i}\right)$ for all $k$, l. This gives us the maps $\psi_{i}$ defined on $\pi\left(U_{i}\right)$ in the desired form. Obviously they are conjugating.

LEMMA 5. If $\nu^{*}$ is a T-invariant Gibbs measure on $\Omega$, then $\nu^{*}(K)=0$, where $K=\bigcup_{k \in \mathbf{Z}} T^{k}(\partial R)$.

Proof. Suppose $\nu^{*}(K)>0$, then so is $\nu^{*}(\partial R)>0$ and thus either $\nu^{*}\left(\bigcap_{i \in \mathbb{N}} T^{i}\left(\partial^{+} R\right)\right)>0$ or $\nu^{*}\left(\bigcap_{i \in \mathbb{N}} T^{-i}\left(\partial^{-} R\right)\right)>0$. Assume the first case holds, and let $K^{*}=\bigcap_{i \in \mathbb{N}} T^{i}\left(\partial^{+} R\right)$. Denote by $B_{\zeta}(z)$ the ball around $z$ with radius $\zeta$. The set $K^{*}$ is invariant under $T$ and is compact; hence there is a point $z \in K^{*}$ such that $\nu^{*}\left(B_{\zeta}(z) \cap K^{*}\right)>0$ for all $\zeta>0$. The points conjugated to $z$ are dense in $\Omega$ and for each of them there exists a conjugating homeomorphism defined in a neighbourhood of $z$ (cf. [2]). So there is a $w \in \Omega$ and a $\delta>0$, so that $B_{2 \delta}(w) \cap K^{*}=\varnothing$, and a conjugating homeomorphism $\psi$ defined on $B_{\zeta}(z)$ for a positive $\zeta$, such that $\psi(z) \epsilon$ $B_{\delta / 2}(w)$ and $\psi\left(B_{\zeta}(z)\right) \subset B_{\delta}(w)$. Set $D=\psi\left(B_{\zeta}(z) \cap K^{*}\right)$, then we have $\nu^{*}(D)>0$, because $\nu^{*}$ is a Gibbs state. Since $\psi$ is conjugating, there exists an $n^{\prime} \in \mathbb{N}$ such that $d\left(T^{i} \circ \psi(y), T^{i}(y)\right)<\delta$ for all $y \in B_{\zeta}(z)$ and $|i| \geq n^{\prime}$. So $\operatorname{dist}\left(T^{i}(D), K^{*}\right)<\delta$, for $K^{*}$ is invariant under $T$. But by construction dist $\left(D, K^{*}\right)>\delta$, hence $T^{i}(D) \cap D=\varnothing$ for all $i \geq n^{\prime}$. The collection $\left\{T^{i n^{\prime}}(D): i \in \mathbb{N}\right\}$ consists of pairwise disjoint sets which have, since $\nu^{*}$ is $T$-invariant, the same positive measure. Hence the measure of their union diverges, which contradicts the normalisation $\nu^{*}(\Omega)=1$.

Proposition 6. For a Gibbs measure $\nu$ on $(\Omega, T)$ we have $\nu(K)=0$.

Proof. Assume $\nu(K)>0$, then there must be an integer $j$ so that $\nu\left(T^{j}(\partial R)\right)>0$ and thus either $\nu\left(T^{j}\left(\partial^{+} R\right)\right)>0$ or $\nu\left(T^{j}\left(\partial^{-} R\right)\right)>0$. Suppose we have the situation $j=0$ and $\nu\left(\partial^{-} R\right)>0$. Then define a sequence of new measures

$$
\nu_{n}=n^{-1} \cdot \sum_{0 \leq i<n} \nu \circ T^{i}
$$

Since $\nu$ is Gibbs it is clear that $\nu \circ T^{i}$ is Gibbs as well for all $i \in \mathbb{Z}$. By the convexity property (cf. [2]) all $\nu_{n}$, for $n \in \mathbb{N}$, are Gibbs measures. Let $\nu^{*}$ be a limit point of $\left\{\nu_{n}: n \in \mathbb{N}\right\}$, and $n[j]$ be a subsequence in $\mathbb{N}$ so that $\nu_{n[j]}$ converges to $\nu^{*}$. For the backward oriented boundary $\partial^{-} R \subset T\left(\partial^{-} R\right)$ holds, and therefore $\nu\left(\partial^{-} R\right) \leq$ $\nu_{n[j]}\left(\partial^{-} R\right)$. We may treat $\partial^{-} R$ as a compact set and have therefore $\lim \sup _{j} \nu_{n[j]}\left(\partial^{-} R\right) \leq \nu^{*}\left(\partial^{-} R\right)$, which is $\leq \nu^{*}(K)=0$ since $\partial^{-} R \subset K$. 
The function $F$, acting on $\Omega$, induces a Hölder continuous real valued function $f=F \circ \pi$ on $\Sigma_{A}$, which is exponentially decreasing with $\alpha=\lambda^{\theta} \in(0,1)$. Let

$$
\operatorname{var}_{k} f=\sup \left\{|f(x)-f(y)|: x, y \in \Sigma_{A} \text { such that } x_{i}=y_{i} \quad \forall|i|<k\right\}
$$

and set

$$
\|f\|=\max \left(\|f\|_{\infty}, \sup _{k \in \mathbf{Z}} \alpha^{-|k|} \operatorname{var}_{k} f\right) .
$$

Restricted to $\Omega \backslash K$, the map $\pi^{-1}$ is one to one; that is, by proposition $6, \pi^{-1}$ is defined $\nu$-almost everywhere. Define the measure $\mu$ on $\Sigma_{A}$ by $\mu(V)=0$ if $V \subset$ $\pi^{-1}(K)$, and $\mu(V)=\mu\left(V \cap \pi^{-1}(\Omega \backslash K)\right)=\nu(\pi(V))$ for all other $V \subset \Sigma_{A}$. Then $\mu$ is a Gibbs state for $f$, since $\nu$ is one for $F$, and all bounded and measurable (test) functions $\tau$ on $\Sigma_{A}$ can be written as $\tau=\tilde{\tau} \circ \pi$ almost everywhere with $\tilde{\tau}$ bounded and measurable functions on $\Omega$.

The cylinder in $\Sigma_{A}$ determined by the string $x_{a}, \ldots, x_{b}$ will be denoted by $U\left(x_{a}, \ldots, x_{b}\right)$ and for convenience we will write $\mu\left(x_{a} \ldots, x_{b}\right)=\mu\left(U\left(x_{a}, \ldots, x_{b}\right)\right)$.

LeMMA 7. Let $f$ be a function on $\Sigma_{A}$ which decreases exponentially fast with $\alpha \in(0,1)$ and let $\sigma$ be topologically mixing. Then there is a constant $C^{*} \in(0, \infty)$ so that

$$
\mu\left(x_{1}, \ldots, x_{m}\right) \cdot \exp \left(m P-\sum_{k \in[1, m]} f \circ \sigma^{k}(x)\right) \in\left[\dot{e}^{-C^{*}}, e^{+C^{*}}\right]
$$

for all $x \in \Sigma_{A}$ and $m \in \mathbb{N}$. The real number $P$ is called the pressure of $f$.

Proof. To verify (1) we will construct a sequence of sets of conjugating homeomorphisms on $\Sigma_{A}$. Since $\sigma$ is supposed to act topologically mixing, we have $A^{n}>0$ if $n$ is large enough. Let $N$ be the smallest such integer and let

$$
\mathscr{T}^{*}[m]=\left\{\left(a_{1}, \ldots, a_{m}\right): a_{i} \in \mathscr{A}, A\left[a_{i}, a_{i+1}\right]=1 \quad \forall i \in[1, m)\right\}
$$

for the set of all $m$-strings. The pressure of $f$ is $P=\lim _{m \rightarrow \infty} P_{m}$, where

$$
P_{m}=m^{-1} \log Z_{m}
$$

and

$$
Z_{m}=\sum_{a \in \mathcal{T}^{*}[m]} \exp \sup \left\{\sum_{k \in[1, m]} f \circ \sigma^{k}(z): z_{i}=a_{i} \quad \forall i \in[1, m]\right\} .
$$

Let us first summon a technical lemma.

LEMMA 8. There exists a number $b$ such that $\left|P_{m}-P\right| \leq b / m$ for all $m \geq 1$.

Proof. Set

$$
\tilde{Z}_{m}=\sum_{z \in \mathscr{P}[m]} \exp \sum_{k \in[1, m]} f \circ \sigma^{k}(z),
$$

where $\mathscr{P}[m]=\left\{x: \sigma^{m}(x)=x, x \in \Sigma_{A}\right\}$ is the set of all $m$-periodic points. Then, using the maximum matrix norm $\|A\|=\max _{i, j}|A[i, j]|$, it follows

$$
\tilde{Z}_{m+N} \leq Z_{m}\left\|A^{N}\right\|^{2} \exp \left(2 N\|f\|_{\infty}\right) \text {. }
$$

Furthermore

$$
Z_{m} \leq \tilde{Z}_{m+N} \exp (2\|f\| /(1-\alpha)) \text {. }
$$

Let $c_{1}=2\|f\|(N+1 /(1-\alpha))+2 \cdot \log \left\|A^{N}\right\|$, then these two inequalities combine to $\left|\log \left(\tilde{Z}_{m+N} Z_{m}^{-1}\right)\right| \leq c_{1}$. As can easily be seen, the pressure may be defined by 
$\lim _{m} m^{-1} \log Z_{m}$ and as well by $\lim _{m} m^{-1} \log \tilde{Z}_{m}$, which is the same. From [4, corollary 7.25] we know that $\left|\log \tilde{Z}_{m}-m P\right| \leq c_{2} t^{m}$ for constants $c_{2}>0$ and $t \in(0,1)$, that is $\left|\log \tilde{Z}_{m+N}-m P\right| \leq c_{2}+N \cdot P$. Now set $b=N \cdot P+c_{1}+c_{2}$.

Recall that $\boldsymbol{A}^{N}>0$ since $\sigma$ is assumed to be topologically mixing. Choose any $x \in \Sigma_{A}$ and $m \in \mathbb{N}$ and fix them throughout the rest of the proof. Now we construct a collection of conjugating maps which are all uniformly continuous and which depend on $x$ and $m$. Set $U=\left\{z: z_{i}=x_{i} \quad \forall i \in[1, m]\right\}$ and choose any string $\omega \in \mathscr{T}^{*}[m]$. Now we define a conjugating map $\varphi$ depending on $\omega$. Set for all $z \in U$ :

(i) $(\varphi(z))_{i}=\omega_{i}$ for all $i \in[1, m]$;

(ii) $(\varphi(z))_{i}=z_{i}$ for all $i \in(-\infty,-N] \cup[m+N,+\infty)$;

(iii) set $U_{\omega}=\left\{z: z_{i}=(\varphi(x))_{i}=\omega_{i} \forall i \in[1, m]\right\}$, and take a covering of $U$ by $|\mathscr{A}|^{2}$ (not necessarily non-empty) sets: $U(s, t)=U \cap\left\{z: z_{-N}=s, z_{m+N}=t\right\}$, for all $s, t \in \mathscr{A}$. The sets $U_{\omega}(s, t)$ are defined analogously. For their measures we obtain

$$
\mu\left(U_{\omega}\right)=\sum_{s, t \in \mathscr{A}} \mu\left(U_{\omega}(s, t)\right)=\sum_{s, t \in \mathscr{A}} \mu\left(\bigcup_{k} U_{\omega}^{k}(s, t)\right),
$$

where $U_{\omega}^{k}(s, t)$ are at most $\left\|A^{N}\right\|^{2}$ disjoint sets of points with the same symbols on the places in $[-N, m+N]$. Altogether they cover $U_{\omega}(s, t)$. Pick out the set $U_{\omega}^{k}(s, t)$ which realises the $\max _{k} \mu\left(U_{\omega}^{k}(s, t)\right)$. This determines the components of $\varphi(\cdot)$ on the places with indices in the two intervals $(-N, 1)$ and $(m, m+N)$.

The map $\varphi: U(s, t) \rightarrow U_{\omega}^{k}(s, t)$ for all $s, t \in \mathscr{A}$ is therefore completely defined. It is clear that $\varphi$ is uniformly continuous on $U(s, t)$ for all $s, t \in \mathscr{A}$, and conjugating, but it is not a homeomorphism. On $U(s, t)$ the map $\varphi$ is finite but at most $\left(|\mathscr{A}| \cdot\left\|A^{N}\right\|\right)^{2}$ to one and may therefore be decomposed in at most $\left(|\mathscr{A}| \cdot\left\|A^{N}\right\|\right)^{2}$ homeomorphisms. Moreover we derive from (2):

$$
\mu(\varphi(U)) \geq\left(|\mathscr{A}| \cdot\left\|A^{N}\right\|\right)^{-2} \mu\left(U_{\omega}\right)
$$

Finally we put

$\mathscr{S}[m]=\left\{\right.$ all $\varphi$ as constructed above with $\omega$ running over the whole $\left.\mathscr{T}^{*}[m]\right\}$.

Let $\mathscr{I}=\{1,2, \ldots,|\mathscr{S}[m]|\}$ be a numbering of $\mathscr{S}[m]$ and label the elements $\varphi_{j}$ in it by an index. Set

$$
g_{j}(x)=\exp \sum_{k \in \mathbf{Z}}\left(f \circ \sigma^{k} \circ \varphi_{j}(x)-f \circ \sigma^{k}(x)\right)
$$

and consider the following weighted sum:

$$
\exp \sum_{k \in[1, m]} f \circ \sigma^{k}(x) \sum_{j \in \Phi}\left\{g_{j}(x) \exp \sum_{k \in(-\infty, 1) \cup(m,+\infty)}-\left(f \circ \sigma^{k} \circ \varphi_{j}(x)-f \circ \sigma^{k}(x)\right)\right\} \text {. }
$$

This is the same as

$$
\begin{aligned}
\sum_{j \in \mathscr{I}} \exp & \sum_{k \in[1, m]} f \circ \sigma^{k} \circ \varphi_{j}(x) \\
= & \sum_{j \in \mathscr{\Phi}} \exp \left(\sup \left\{\sum_{k \in[1, m]} f \circ \sigma^{k}(z): z_{i}=\left(\varphi_{j}(x)\right)_{i} \quad \forall i \in[1, m]\right\}+r_{j}\right),
\end{aligned}
$$


where the remainders $r_{j}$ are estimated as $\left|r_{j}\right| \leq\|f\|\left(1+2 \alpha\left(1-\alpha^{m}\right) /(1-\alpha)\right)$. The sum (4) lies therefore in the interval $Z_{m}\left[e^{-c}, e^{c}\right]$, where $c=\|f\|(1+2 /(1-\alpha))$. Let $P_{m}=$ $m^{-1} \log Z_{m}$ and set

$$
\theta_{m}=\mu\left(x_{1}, \ldots, x_{m}\right) \cdot \exp \left(m P_{m}-\sum_{k \in[1, m]} f \circ \sigma^{k}(x)\right) .
$$

Observe that replacing $P_{m}$ by $P$ transforms $\theta_{m}$ into the expression on the left of (1). We use (4) to get rid of the factor $m P_{m}$ in the exponential. Instead we get a summation over $j$, expressing the sum over all $m$-strings in the definition of the pressure. So we end up with

$$
\theta_{m}=\mu\left(x_{1}, \ldots, x_{m}\right) d_{1} \sum_{j \in \Phi} d_{2, j} g_{j}(x),
$$

where $d_{1} \in\left[e^{-c}, e^{c}\right]$, and

$$
d_{2, j}=\exp \sum_{k \in(-\infty, 1) \cup(m,+\infty)}\left(f \circ \sigma^{k} \circ \varphi_{j}(x)-f \circ \sigma^{k}(x)\right) \in\left[e^{-c^{\prime}}, e^{c^{\prime}}\right]
$$

for all $j \in \mathscr{I}$, with $c^{\prime}=2 /(1-\alpha)$. For all $y \in U$ and $j \in \mathscr{I}$ we estimate $\left|\log \left(g_{j}(x) g_{j}(y)^{-1}\right)\right|$

$$
\begin{aligned}
& \leq \sum_{k \in(-\infty, 1) \cup(m,+\infty)}\left\{\left|f \circ \sigma^{k} \circ \varphi_{j}(x)-f \circ \sigma^{k}(x)\right|+\left|f \circ \sigma^{k} \circ \varphi_{j}(y)-f \circ \sigma^{k}(y)\right|\right\} \\
& \quad+\sum_{k \in[1, m]}\left\{\left|f \circ \sigma^{k} \circ \varphi_{j}(x)-f \circ \sigma^{k} \circ \varphi_{j}(y)\right|+\left|f \circ \sigma^{k}(x)-f \circ \sigma^{k}(y)\right|\right\} \\
& \leq 2\|f\|\left(N+2 /(1-\alpha)+1+2 \alpha\left(1-\alpha^{m}\right) /(1-\alpha)\right) .
\end{aligned}
$$

Set $c^{\prime \prime}=2\|f\|(N+1+4 /(1-\alpha))$ and it simplifies to

$$
\left|\log \left(g_{j}(x) g_{j}(y)^{-1}\right)\right| \leq c^{\prime \prime}
$$

Define the characteristic function of $U$

$$
\tau_{m}^{x}(y)= \begin{cases}1 & \text { if } y_{i}=x_{i} \text { for all } i \in[1, m] \\ 0 & \text { otherwise. }\end{cases}
$$

Then we have $U=\left\{y: y \in \Sigma_{A}, \tau_{m}^{x}(y)=1\right\}$. For $\theta_{m}$ we may write

$$
\theta_{m}=\sum_{j \in \mathscr{S}} d_{1} d_{2, j} g_{j}(x) \int_{U} \tau_{m}^{x} d \mu \in \sum_{j \in \Phi} \int_{U} g_{j} \tau_{m}^{x} d \mu\left[e^{-c-c^{\prime}-c^{\prime \prime}}, e^{c+c^{\prime}+c^{\prime \prime}}\right]
$$

- the last step following from inequality (5). To evaluate the integral on the right, we remember $\mu$ is a Gibbs state for $f$. According to the note made when defining the conjugating maps, we decompose, for every $j$, the cylinder $U$ into a finite number of sets $U[k, j]$, i.e. $U=\bigcup_{k \in \mathscr{F}[j]} U[k, j]$, where $\left.\varphi_{j}\right|_{U[k, j]}$ are homeomorphisms and $\mathscr{J}[j] \leq\left(|\mathscr{A}| \cdot\left\|A^{N}\right\|\right)^{2}$ for all $j$. Hence

$$
\begin{aligned}
\sum_{j \in \mathscr{I}} \int_{U} g_{j} \cdot \tau_{m}^{x} d \mu & =\sum_{j \in \mathscr{I}} \sum_{k \in \mathscr{F}[j]} \int_{U[k, j]} g_{j} \cdot \tau_{m}^{x} d \mu \\
& =\sum_{j \in \mathscr{I}} \sum_{k \in \mathscr{I}[j]} \int \tau_{m}^{x} \circ \varphi_{j}^{-1} d \mu,
\end{aligned}
$$


where the integrals in the last line are taken over $\varphi_{j}(U[k, j])$. This expression lies in the interval $\left[\left(|\mathscr{A}| \cdot\left\|A^{N}\right\|\right)^{-2},\left(|\mathscr{A}| \cdot\left\|A^{N}\right\|\right)^{2}\right]$. To see this, observe that the set

$$
\left\{\omega[j]: \omega[j]=\left(\left(\varphi_{j}(x)\right)_{1}, \ldots,\left(\varphi_{j}(x)\right)_{m}\right), j \in \mathscr{I}\right\}
$$

is just $\mathscr{T}^{*}[m]$, that is $\Sigma_{A}=\bigcup_{j \in \mathscr{I}} U_{\omega[j]}$. But $\bigcup_{j \in \mathscr{T}} \varphi_{j}(U)$ does not cover the whole space $\Sigma_{A}$, since there was a choice in (iii) in defining $\varphi_{j}$. This with inequality (3) provides the lower bound. The upper can be explained by the possibility that the maps $\varphi_{j}$ have to be decomposed in $|\mathscr{F}[j]| \leq\left(|\mathscr{A}| \cdot\left\|A^{N}\right\|\right)^{2}$ homeomorphisms. It is therefore proved that $\theta_{m} \in\left[e^{-C}, e^{C}\right]$ with $C=c+c^{\prime}+c^{\prime \prime}+2 \cdot \log \left(\left\|A^{N}\right\| \cdot|A| A \mid\right)$ a constant independent of $x \in \Sigma_{A}$ and $m \in \mathbb{N}$. Finally we replace $P_{m}$ by $P$, which is compensated for by increasing $C$ to $C+b$, with the same $b$ as in lemma 8 . Set $C^{*}=C+b$ and (1), and hence lemma 9 , is proved for all $x \in \Sigma_{A}$ and $m \in \mathbb{N}$.

LEMMA 9. The wandering set has measure zero.

Proof. The lemma is proved for the shift space and clearly holds then in the case of the underlying Smale space as well. Let $A$ be the transition matrix. It may be put in the form

$$
\left|\begin{array}{cccc}
A_{1,1} & A_{1,2} & \cdots & A_{1, t} \\
0 & A_{2,2} & \cdots & \\
& & & \vdots \\
0 & \cdots & 0 & A_{t, t}
\end{array}\right|,
$$

where $A_{i, i}$ are irreducible $r_{i} \times r_{i}$-matrices. The alphabet $\mathscr{A}$ splits into $\left(\mathscr{A}_{1}, \ldots, \mathscr{A}_{t}\right)$, with $\left|\mathscr{A}_{i}\right|=r_{i}$ for all $i$. The subshifts $\Sigma_{\mathrm{i}}$ generated by $A_{i, i}$ over $\mathscr{A}_{i}$ are topologically transitive and decompose into finitely many subshifts $\Sigma_{j, q}$ on which a power $\sigma^{s}$ of the shift acts topologically mixing. We renumber the shifts $\Sigma_{i, q}$ as $\Sigma_{i}$ for $i \in\left[1, t^{\prime}\right]$ with $t^{\prime} \geq t$. Let $\sigma^{s}$ for convenience now be called $\sigma$ and let $N$ be the smallest number so that $A_{i, i}^{N}>0$ for all $i \in\left[1, t^{\prime}\right]$ (with the new $A_{i, i}$ ) and denote by $P[i]$ the pressure of $f$ on $\Sigma_{i}$. Points $x=\left(x_{k}\right)_{k \in \mathbb{Z}}$ in the wandering set of $\Sigma_{A}$ are sequences with elements $x_{k}$ not all in one sub-alphabet $\mathscr{A}_{i}$ but in several; however the indices $i[k]$ of $\mathscr{A}_{i[k]}$ to which the $x_{k}$ belong never decrease as $k$ increases. Suppose the wandering set has positive measure, then there exists a subset $U(\zeta) \subset \Sigma_{A}, \zeta=\zeta_{a}, \ldots, \zeta_{b}$ with positive measure and for which $U(\zeta) \cap U\left(\sigma^{m}(\zeta)\right)=\varnothing$ for all $m \in \mathbb{Z} \backslash\{0\}$, where $U(\zeta)$ denotes the cylinder in $\Sigma_{A}$ which is determined by the string $\zeta$.

Consider the cover $U(\zeta)=\bigcup U\left(\zeta_{a-1}, \zeta\right)$, where $\zeta_{a-1}$ runs over all symbols with $A\left[\zeta_{a-1}, \zeta_{a}\right]=1$. If $\zeta_{a} \in \mathscr{A}_{i}$ and $\zeta_{a-1} \in \mathscr{A}_{i^{\prime}}$ for $i<i^{\prime}$ and if $\mu\left(\zeta_{a-1}, \zeta\right)>0$ then we replace $\zeta=\zeta[0]$ by $\zeta[1]=\zeta_{a-1}, \zeta$ (again $\mu\left(\zeta_{a-1}, \zeta\right)$ stands for $\mu\left(U\left(\zeta_{a-1}, \zeta\right)\right)$ ); otherwise we use for $\zeta_{a-1}$ any other symbol such that $\mu\left(\zeta_{a-1}, \zeta\right)>0$. Repeat this procedure for $\zeta_{b+1}$ and increase the index of the sub-alphabet if possible or leave it the same. In that way we get $\zeta[2]=\zeta[1], \zeta_{b+1}$ with $\zeta_{b} \in \mathscr{A}_{j}$ and $\zeta_{b+1} \in \mathscr{A}_{j^{\prime}}$, where $j \leq j^{\prime}$. Iterating this process one obtains a sequence of strings $\zeta[i]$ of increasing length and $\mu(\zeta[i])>0$ for $i \in \mathbb{N} \cup\{0\}$. At any stage the elements on both sides must remain in the same sub-alphabets; that is, there exists a number $M>\max (|\mathrm{a}|,|b|)$ and $i, j$ with $1 \leq i<j \leq$ $t$, so that $\zeta_{-k} \in \mathscr{A}_{i}$ and $\zeta_{k} \in \mathscr{A}_{j}$ for all $k \geq M$. Set $\zeta_{-M}, \ldots \zeta_{M}=\xi$ and $\tilde{U}(\xi)=\left\{x: x_{-k} \in \mathscr{A}_{i}, x_{k} \in \mathscr{A}_{j}\right.$, for $k>M$,

$$
\left.x_{k}=\xi_{k} \text { for }|k| \leq M \text { and } A\left[x_{k}, x_{k+1}\right]=1 \text { for all } k \in \mathbb{Z}\right\}
$$


The same notation will be used whenever there are cylinders with elements on the negative side restricted to $\mathscr{A}_{i}$ (respectively $\mathscr{A}_{j}$ on the positive side). By construction $\mu(\tilde{U}(\xi))>0$ and

$$
\sigma^{m}(\tilde{U}(\xi)) \cap \tilde{U}(\xi)=\varnothing
$$

holds true for all $m \in \mathbb{Z} \backslash\{0\}$. Suppose $P[i] \geq P[j]$. Fix $m>2 M+1+N$ and denote by $\{\gamma[p]: p \in[1, h]\}$, for a number $h$, the set of all $m$-strings in $\Sigma_{i}$. Let us construct conjugating homeomorphisms $\left\{\varphi_{p}: p \in[1, h]\right\}$. Select an $m$-string $\theta$ in $\Sigma_{j}$, then $\mu(\tilde{U}(\xi, \ldots, \theta))$ is positive, for all choices of $\theta$ since $\mu$ is Gibbs. The dots denote room for $N-1$ symbols. Decompose

$$
\tilde{U}(\xi, \ldots, \theta)=\bigcup_{\eta, \eta^{\prime}} \tilde{U}\left(\eta, \xi, \eta^{\prime}, \theta\right),
$$

where $\eta$ runs over all $(2 N-1)$-strings in $\Sigma_{i}$ and $\eta^{\prime}$ over all $(N-1)$-strings in $\Sigma_{j}$. Select a pair $\left(\eta, \eta^{\prime}\right)$ so that

$$
\mu\left(\tilde{U}\left(\eta, \xi, \eta^{\prime}, \theta\right)\right) \geq c_{0}^{-1} \mu(\tilde{U}(\xi, \ldots, \theta)),
$$

with $c_{0}=|\mathscr{A}| \cdot\left\|A^{N}\right\| \cdot\left\|A^{2 N}\right\|$.

Define $\varphi_{p}$ for $p \leq \not$ and set for $z \in \tilde{U}\left(\eta, \xi, \eta^{\prime}, \theta\right)$ :

(i) $\left(\varphi_{p}(z)\right)_{k}=z_{k}$ for $k \in(-\infty,-M-2 N] \cup[M+N+m, \infty)$,

(ii) $\left(\varphi_{p}(z)\right)_{k}=z_{k-m}$ for $k \in[-M+m, M+m]$,

(iii) $\left(\left(\varphi_{p}(z)\right)_{-M-N}, \ldots,\left(\varphi_{p}(z)\right)_{-M-N+m}\right)=\gamma[p]$,

(iv) something fitting up in $(-M-2 N,-M-N),(-M-N+m,-M+m)$ and $(M+m, M+N+m)$, which will be specified shortly.

Thus it follows for the Radon-Nykodym derivative

$$
\begin{aligned}
\log d \varphi_{p} \mu / d \mu(z)= & \sum_{k \in \mathbf{Z}}\left(f \circ \sigma^{k} \circ \varphi_{p}(z)-f \circ \sigma^{k}(z)\right) \\
= & \sum_{k \in(-\infty,-M-2 N] \cup[M+N+m, \infty)}\left(f \circ \sigma^{k} \circ \varphi_{p}(z)-f \circ \sigma^{k}(z)\right) \\
& +\sum_{k \in[-M, M]}\left(f \circ \sigma^{k+m} \circ \varphi_{p}(z)-f \circ \sigma^{k}(z)\right. \\
& +\sum_{k \in(-M-2 N,-M-N) \cup(-M-N+m,-M+m) \cup(M+m, M+N+m)} f \circ \sigma^{k} \circ \varphi_{p}(z) \\
& \left.-\sum_{k \in(-M-2 N,-M) \cup(M, M+N)} f \circ \sigma^{k}(z)\right) \\
& +\sum_{k \in[-M-N,-M-N+m]}\left(f \circ \sigma^{k} \circ \varphi_{p}(z)-f \circ \sigma^{k+2(M+N)}(z)\right) .
\end{aligned}
$$

The first and second of these four sums are in modulus less than $4\|f\| /(1-\alpha)$, and the third and fourth together are less than $3\|f\|(N+4 /(1-\alpha))$. Put $c=$ $3\|f\|(N+8 /(1-\alpha))$. By construction we made the sets $\varphi_{p}\left(\tilde{U}\left(\eta, \xi, \eta^{\prime}, \theta\right)\right)$ pairwise disjoint, and furthermore

$$
\varphi_{p}\left(\tilde{U}\left(\eta, \xi, \eta^{\prime}, \theta\right)\right) \subset \sigma^{m}(\tilde{U}(\xi)),
$$

that is, for different $m$ we get disjoint sets. As pointed out in lemma 7 (iii), the fitting-up strings in (iv) may be chosen so that

$$
\mu\left(\sigma^{m}(\tilde{U}(\xi))\right) \geq c_{1}^{-1} \mu\left(\bigcup_{p \in[1, k]} \varphi_{p}\left(\tilde{U}\left(\eta, \xi, \eta^{\prime}, \theta\right)\right)\right),
$$


with $c_{1}=|\mathscr{A}|^{2} \cdot\left\|A^{N}\right\|^{3}$. So we have for any $z \in \tilde{U}\left(\eta, \xi, \eta^{\prime}, \theta\right)$ :

$$
\begin{aligned}
\mu\left(\sigma^{m}(\tilde{U}(\xi))\right) \geq & c_{1}^{-1} e^{-c} \mu\left(\tilde{U}\left(\eta, \xi, \eta^{\prime}, \theta\right)\right) \\
& \times \sum_{p \in[1, k]} \exp \sum_{k \in[1, m]}\left(f \circ \sigma^{k-M-N} \circ \varphi_{p}(z)-f \circ \sigma^{k+M+N}(z)\right) \\
\geq & \left.c_{1}^{-1} e^{-c-P[j]} \mu\left(\tilde{U}\left(\eta, \xi, \eta^{\prime}, \theta\right)\right) \exp \left\{P[j]-\sum_{k \in[1, m]} f \circ \sigma^{k+M+N}(z)\right)\right\} \\
& \times \sum_{p \in[1, k]} \exp \sum_{k \in[1, m]} f \circ \sigma^{k-M-N} \circ \varphi_{p}(z)
\end{aligned}
$$

The same argument as in lemma 7 provides a more general form of the inequalities (1), namely

$$
\left.\mu(\tilde{U}(\xi, \ldots, \theta)) \exp \left\{m P[j]-\sum_{k \in[1, m]} f \circ \sigma^{k+M+N}(z)\right)\right\} \in \mu(\tilde{U}(\xi))\left[e^{-C^{*}}, e^{+C^{*}}\right],
$$

for $z \in \tilde{U}(\xi, \ldots, \theta)$. To see this, observe that the conjugating homeomorphisms there are to be constructed in exactly the same way. The result for Zeta-functions involving periodic points, which is cited in lemma 8 , works the same in this case too, since the number of periodic points with period $h$ is the trace of the $h$ th power of the transition matrix, which here is $\operatorname{tr}\left(A_{j, j}^{h}\right)$. To evaluate the pressure in that case we choose only points which are periodic in the components with index $\geq M+N$.

By following the argument after inequality (4) the summation over $p \in[1, h]$ gives rise to a factor $\exp \left(m P_{m}[i]\right)$. Using (6) we end up with

$$
\mu\left(\sigma^{m}(\tilde{U}(\xi))\right) \geq c_{0}^{-1} c_{1}^{-1} e^{-c-C^{*}} d_{1} \mu(\tilde{U}(\xi)) \exp \left[m\left(P_{m}[i]-P[j]\right)\right],
$$

where $d_{1}$ is the same constant as in lemma 7 . Finally, again using lemma 8 , this transforms to

$$
\begin{aligned}
\mu\left(\sigma^{m}(\tilde{U}(\xi))\right) & \geq c_{0}^{-1} c_{1}^{-1} e^{-c-C^{*}-b} d_{1} \mu(\tilde{U}(\xi)) \exp [m(P[i]-P[j])] \\
& \geq c_{0}^{-1} c_{1}^{-1} e^{-c-C^{*}-b} d_{1} \mu(\tilde{U}(\xi)),
\end{aligned}
$$

since we supposed $P[i]-P[j]$ not to be negative. Summing up over $m$ gives a contradiction to the normalisation condition $\mu\left(\Sigma_{A}\right)=1$, hence $\mu(\tilde{U}(\xi))=\mu(\zeta)=0$, and therefore the lemma follows, since the wandering set of $\left(\Sigma_{A}, \sigma\right)$ is contained in the one of $\left(\Sigma_{A}, \sigma^{s}\right)$. If $P[i]-P[j]$ happens to be negative we construct the conjugating homeomorphisms replacing $m$ by $-m$ and then get the lower bound for $\mu\left(\sigma^{m}(\tilde{U}(\xi))\right)$ in the same way as described.

LEMMA 10. Let $\mu$ be a Gibbs state on $\left(\Sigma_{A}, \sigma\right)$, then it is $\sigma$-invariant if $\sigma$ acts topologically mixing.

Proof. Let $\mathscr{T}[b-a]$ be the set consisting of all possible strings $x_{a}, \ldots, x_{b}$ of length $b-a$ for $a, b \in \mathbb{Z}$ and $a \leq b$.

Assume $\mu$ not to be $\sigma$-invariant, then there exists a set $B \subset \Sigma_{A}$ such that $\mid \mu(\sigma(B))-$ $\mu(B) \mid>0$ and $\mu(B)>0$. Suppose it is $\mu(\sigma(B)) \geq \rho \cdot \mu(B)$ for a number $\rho>1$. By a covering argument we conclude that there must be a cylinder $U(\zeta)$ determined by the string $\zeta=x_{a}, \ldots, \mathbf{x}_{0}, \ldots, x_{b}$ (bold characters denote the zero position), such that

$$
\mu\left(\zeta^{*}\right) \geq \rho \cdot \mu(\zeta),
$$

where $\zeta^{*}=x_{a}, \ldots, \mathbf{x}_{1}, \ldots, x_{b}$ is the shifted string $\zeta$. A partial covering of $\Sigma_{A}$ will 
now be constructed, and then it will be shown that $\mu\left(\Sigma_{A}\right) \geq \sqrt{ } \sqrt{ } \rho$, which contradicts the normalisation of $\mu$.

Let $\tau$ be an even number such that

$$
16\|f\| \cdot \alpha^{\tau / 2} \leq \log \rho,
$$

and let us construct a cover of $U(\zeta)$ by cylinder sets of the form $U\left(\beta, \eta, \zeta, \eta^{\prime}, \beta^{\prime}\right)$, where $\beta, \beta^{\prime} \in \mathscr{T}[\tau], \eta, \eta^{\prime} \in \mathscr{T}[N-1]$. There are strings $\beta, \beta^{\prime}, \eta, \eta^{\prime}$ so that

$$
\mu\left(\beta, \eta, \zeta^{*}, \eta^{\prime}, \beta^{\prime}\right) \geq \rho \cdot \mu\left(\beta, \eta, \zeta, \eta^{\prime}, \beta^{\prime}\right)
$$

One single ${ }^{*}$ means the whole string is to be shifted. For the moment fix $\beta, \beta^{\prime}, \eta$, $\eta^{\prime}$, and proceed to construct a pair of conjugating homeomorphisms $\varphi, \varphi^{\prime}$ as follows. Choose any string $\omega \in \mathscr{T}[b-a]$ with $\omega=\omega_{a}, \ldots, \omega_{0}, \ldots, \omega_{b}$ and define for all $z \in U\left(\beta, \eta, \zeta, \eta^{\prime}, \beta^{\prime}\right):$

(i) $(\varphi(z))_{i}=\omega_{i}$ for all $i \in[a, b]$;

(ii) $(\varphi(z))_{i}=z_{i}$ for all $i \in(-\infty, a-N] \cup[b+N, \infty)$;

(iii) any strings $\theta, \theta^{\prime} \in \mathscr{T}[N-1]$ to join up the ends in the intervals $(a-N, a)$ and $(b, b+N)$.

Secondly we define $\varphi^{\prime}$ on the cylinder $U\left(\beta, \eta, \zeta^{*}, \eta^{\prime}, \beta^{\prime}\right)$ and set for all $z$ in it

(i') $\left(\varphi^{\prime}(z)\right)_{i}=\left(\omega^{*}\right)_{i}$ for all $i+1 \in[a, b]$;

(ii') $\left(\varphi^{\prime}(z)\right)_{i}=z_{i}$ for all $i+1 \in(-\infty, a-N] \cup[b+N, \infty)$;

(iii') the same strings $\theta$ and $\theta^{\prime}$ as in (iii) to fill the two gaps.

We observe that $\varphi^{\prime} \circ \sigma \circ \varphi^{-1}=\sigma$ on $U\left(\beta, \theta, \omega, \theta^{\prime}, \beta^{\prime}\right)$. Denote by $\chi\left(x_{a}, \ldots, \mathbf{x}_{0}, \ldots, x_{b}\right)$ the characteristic function of $U\left(x_{a}, \ldots, \mathbf{x}_{0}, \ldots, x_{b}\right)$. Since $\mu$ is by hypothesis a Gibbs state we conclude for any $x \in U\left(\beta, \eta, \zeta, \eta^{\prime}, \beta^{\prime}\right)$ :

$$
\begin{aligned}
\mu\left((\varphi(x))_{a-N-\tau}, \ldots,(\varphi(\mathbf{x}))_{0}, \ldots,(\varphi(x))_{b+N+\tau}\right) \\
=\int \chi\left((\varphi(x))_{a-N-\tau}, \ldots,(\varphi(\mathbf{x}))_{0}, \ldots,(\varphi(x))_{b+N+\tau}\right) d \mu \\
=\int \chi\left((\varphi(x))_{a-N-\tau}, \ldots,(\varphi(\mathbf{x}))_{0}, \ldots,(\varphi(x))_{b+N+\tau}\right) \\
\circ \varphi \exp \sum_{k \in \mathbf{Z}}-\left(f \circ \sigma^{k} \circ \varphi-f \circ \sigma^{k}\right) d \mu .
\end{aligned}
$$

This leads to

$$
\mu\left(\beta, \theta, \omega, \theta^{\prime}, \beta^{\prime}\right) \geq \mu\left(\beta, \eta, \zeta, \eta^{\prime}, \beta^{\prime}\right) \exp \left\{\sum_{k \in \mathbf{Z}}-\left(f \circ \sigma^{k} \circ \varphi(x)-f \circ \sigma^{k}(x)\right)-c\right\},
$$

where

$$
c=4\|f\| \cdot \alpha^{\tau / 2} .
$$

In the last estimate we made use of the fact that

$$
z_{i}=x_{i}=(\varphi(x))_{i}
$$

for all $z \in U\left(\beta, \theta, \zeta, \theta^{\prime}, \beta^{\prime}\right)$ and $i \in[a-N-\tau, a-N] \cup[b+N, b+N+\tau]$. The same estimate holds on the shifted sets using $\varphi^{\prime}$. For any $y \in U\left(\beta, \eta, \zeta^{*}, \eta^{\prime}, \beta^{\prime}\right)$,

$$
\mu\left(\beta, \theta, \omega^{*}, \theta^{\prime}, \beta^{\prime}\right) \geq \mu\left(\beta, \eta, \zeta^{*}, \eta^{\prime}, \beta^{\prime}\right) \exp \left\{\sum_{k \in \mathbf{Z}}-\left(f \circ \sigma^{k} \circ \varphi^{\prime}(y)-f \circ \sigma^{k}(y)\right)-c\right\}
$$


holds true. Set $y=\sigma(x)$ and (7) and (8) combine to give

$$
\begin{array}{r}
\mu\left(\beta, \theta, \omega^{*}, \theta^{\prime}, \beta^{\prime}\right) \geq \rho \cdot \mu\left(\beta, \theta, \omega, \theta^{\prime}, \beta^{\prime}\right) \exp \left\{\sum _ { k \in \mathbb { Z } } \left[\left(f \circ \sigma^{k} \circ \varphi(x)-f \circ \sigma^{k}(x)\right)\right.\right. \\
\left.\left.-\left(f \circ \sigma^{k} \circ \varphi^{\prime} \circ \sigma(x)-f \circ \sigma^{k} \circ \sigma(x)\right)\right]-2 c\right\} .
\end{array}
$$

Set $M^{*}=\max (|a-N|,|b+N|)$, choose $M>M^{*}$ and estimate the sum in the exponential by

$$
\begin{aligned}
& \leq \sum_{|k| \geq M}\left\{\left|f \circ \sigma^{k} \circ \varphi^{\prime} \circ \sigma(x)-f \circ \sigma^{k+1}(x)\right|+\left|f \circ \sigma^{k} \circ \varphi(x)-f \circ \sigma^{k}(x)\right|\right\} \\
& \quad+\mid \sum_{|k|<M}\left\{-\left(f \circ \sigma^{k} \circ \varphi^{\prime} \circ \sigma(x)-f \circ \sigma^{k} \circ \varphi(x)\right\}+f \circ \sigma^{M}(x)-f \circ \sigma^{1-M}(x) \mid\right.
\end{aligned}
$$

and since we identify $\sigma \circ \varphi(x)$ with $\varphi^{\prime} \circ \sigma(x)$, we have

$$
\begin{aligned}
& \leq 2\|f\| \cdot \alpha^{M+\tau-M^{*}} /(1-\alpha)+\mid-f \circ \sigma^{M-1} \circ \varphi^{\prime} \circ \sigma(x)+f \circ \sigma^{1-M} \circ \varphi(x)+f \circ \sigma^{M}(x) \\
& \quad-f \circ \sigma^{1-M}(x) \mid \\
& \leq 2\|f\| \cdot\left(\alpha^{M+\tau-M^{*}} /(1-\alpha)+\alpha^{M-M^{*}-1}\right) .
\end{aligned}
$$

This tends to zero as $M$ tends to infinity. By the choice of $\tau$ we obtain

$$
\mu\left(\beta, \theta, \omega^{*}, \theta^{\prime}, \beta^{\prime}\right) \geq \sqrt{ } \rho \cdot \mu\left(\beta, \theta, \omega, \theta^{\prime}, \beta^{\prime}\right)
$$

for all $\omega \in \mathscr{T}[b-a]$ and suitable $\theta, \theta^{\prime} \in \mathscr{T}[N-1]$. The strings $\beta$ and $\beta^{\prime}$ are determined by the point $x \in U\left(\beta, \theta, \zeta, \theta^{\prime}, \beta^{\prime}\right)$, and therefore only a part of $\Sigma_{A}$ gets covered by varying $\omega$ over the whole $\mathscr{T}[b-a]$. Now we proceed to cover in the second generation, whereby we partly cover the complement of what was already covered in the first step. Let the strings $\beta, \eta, \zeta$ etc. now be given an index $1\left(\beta_{1}, \eta_{1}, \zeta_{1}\right.$ etc. $)$ and set $\zeta_{2}=\beta_{1}, \eta_{1}, \zeta_{1}, \eta_{1}^{\prime}, \beta_{1}^{\prime}$. The cylinder $U\left(\zeta_{2}\right)$ now gets covered by smaller ones, and again there is at least one cylinder for which

$$
\mu\left(\beta_{2}, \eta_{2}, \zeta_{2}^{*}, \eta_{2}^{\prime}, \beta_{2}^{\prime}\right) \geq \rho \cdot \mu\left(\beta_{2}, \eta_{2}, \zeta_{2}, \eta_{2}^{\prime}, \beta_{2}^{\prime}\right),
$$

where $\beta_{2}, \beta_{2}^{\prime} \in \mathscr{T}[\tau]$ and $\eta_{2}, \eta_{2}^{\prime} \in \mathscr{T}[N-1]$. For the second generation $\omega_{2}, \theta_{2}, \theta_{2}^{\prime}$ we proceed as above. Call the union of all cylinders constructed in the $i$ th generation $V_{i}$ for $i \in \mathbb{N}$, then we have $V_{i} \cap V_{j}=\varnothing$ if $i \neq j$. It is possible to cover in this manner arbitrarily large subsets of $\Sigma_{A}$. To make it obvious we will show that

$$
\mu\left(\mathrm{c}\left(\bigcup_{1 \leq i \leq n} V_{i}\right)\right) \rightarrow 0
$$

for $n \rightarrow \infty$, where $\mathbf{C}$ denotes the complement in $\Sigma_{A}$. In constructing the cylinders on which $\varphi$ (respectively $\left.\varphi^{\prime}\right)$ are defined we keep fixed the $(\tau+N)$-strings at each end of $\omega$, and thus we select just one small cylinder from at most $\left(|\mathscr{A}| \cdot\left\|A^{\tau+N}\right\|\right)^{2}$ small cylinders. Their $\mu$-measures may be compared, for example by constructing conjugating homeomorphisms. Since $\mu$ is Gibbs, the ratio of two of them is at most

$$
\exp [2 \cdot\|f\|(\tau+N+2 /(1-\alpha))] \text {. }
$$

This allows us to deduce a lower bound for the measure of $V_{n}$ for $n \geq 1$, depending on all the previous generations these subsets. If we set

$$
\Xi=\left(|\mathscr{A}| \cdot\left\|A^{\tau+N}\right\|\right)^{-2} \exp [-2 \cdot\|f\|(\tau+N+2 /(1-\alpha))],
$$


which is a positive constant, we conclude that

$$
\mu\left(V_{n}\right) \geq \Xi \cdot \mu\left(\mathbf{C}\left(\bigcup_{1 \leq i<n} V_{i}\right)\right),
$$

for all $n \in \mathbb{N}$. Since the $V_{i}$ are pairwise disjoint, we have

$$
\begin{aligned}
\mu\left(\mathrm{C}\left(\bigcup_{1 \leq i \leq n} V_{i}\right)\right) & =\mu\left(\mathbf{C}\left(\bigcup_{1 \leq i<n} V_{i}\right)\right)-\mu\left(V_{n}\right) \\
& \leq(1-\Xi) \mu\left(\mathbf{C}\left(\bigcup_{1 \leq i<n} V_{i}\right)\right)
\end{aligned}
$$

Iteration yields

$$
\mu\left(\mathrm{C}\left(\bigcup_{1 \leq i \leq n} V_{i}\right)\right) \leq \mu\left(\mathrm{C} V_{1}\right)(1-\Xi)^{n-1}
$$

and this tends to zero as $n$ tends to infinity. By construction it is

$$
\mu\left(\sigma\left(V_{i}\right)\right) \geq \sqrt{ } \rho \cdot \mu\left(V_{i}\right)
$$

for all $i \in \mathbb{N}$, and hence

$$
\mu\left(\sigma\left(\Sigma_{A}\right)\right) \geq \mu\left(\sigma\left(\bigcup_{1 \leq i \leq n} V_{i}\right)\right) \geq \sqrt{ } \sqrt{ } \rho>1
$$

if $n$ is large enough. This is impossible. In the case where $\mu(\sigma(B)) \leq \rho \cdot \mu(B)$ for $\rho \in(0,1)$ we replace $\sigma$ by $\sigma^{-1}, \rho$ by $\rho^{-1}$ and proceed in the same way.

Proposition 11. Let $f$ be a function on $\Sigma_{A}$ which decreases exponentially fast with $\alpha \in(0,1)$ and let $\sigma$ be topologically mixing. If $\mu$ is a Gibbs state for $f$ then it is also an equilibrium state.

Proof. The proof is a reference to [1, Theorems 1.2 and 1.22]. In lemma 7 we have checked the conditions for a Gibbs state in Bowen's sense. Lemma 10 shows $\sigma$-invariance of $\mu$ and thus it is an equilibrium state for $f$ on $\left(\Sigma_{A}, \sigma\right)$. Since $f$ is Hölder continuous it is the unique one.

LemMa 12. Suppose we have a Smale space where a homeomorphism acting topologically mixing implies that a Gibbs state for a given function has to be an equilibrium state.

Let $\nu$ be any Gibbs state. Then there exists a number $s \in \mathbb{N}$, such that $\nu$ is an equilibrium state for $F_{s}=\sum_{r \in[0, s)} F \circ T^{r}$ on $\left(\Omega, T^{s}\right)$.

Proof. The proof is by Smale's spectral decomposition (cf. [1, theorem 3.5]). The non-wandering set of $\Omega$ is a union of finitely many disjoint compact sets $\Omega^{v}$, called basic sets, which are invariant under $T$ and on which $T$ acts topologically transitively. Points which are conjugated always lie in the same basic set $\Omega^{v}$, each of which is itself a union of $t[v]$ many disjoint, compact sets $\Omega^{v, u}$ on which $T^{t[v]}$ acts topologically mixing and where we have $T^{t[v]}\left(\Omega^{v, u}\right)=\Omega^{v, u}$, for all $u \in[1, t[v]]$. Each set $\Omega^{v, u}$ has positive distance from all the others, larger than $\delta$, say. For any conjugating homeomorphism $\psi$ defined on $U \subset \Omega$ there is

$$
d\left(T^{k i[v]} \circ \psi(z), T^{k t[v]}(z)\right)<\delta,
$$

for all $z \in U$ and where $|k|$ is big enough. That is, $\psi$ restricted to $\bar{U} \cap \Omega^{v, u}$ again maps into $\Omega^{v, u}$. So we restrict to maps $\psi$ acting only on $\Omega^{v, u}$, replace $T$ by $T^{t[v]}$ and 
$F$ by $F_{v}=\sum_{0 \leq r<t[v]} F \circ T^{r}$; then

$$
\begin{aligned}
\log g & =\sum_{i \in \mathbf{Z}}\left(F \circ T^{i} \circ \psi-F \circ T^{i}\right) \\
& =\sum_{i \in \mathbf{Z}} \sum_{0 \leq r<r[v]}\left(F \circ T^{r+i t[v]} \circ \psi-F \circ T^{r+i t[v]}\right) \\
& =\sum_{i \in \mathbf{Z}}\left(F_{v} \circ T^{i t[v]} \circ \psi-F_{v} \circ T^{i t[v]}\right) .
\end{aligned}
$$

If $\nu$ is a Gibbs state for $F$ on $(\Omega, T)$ then for each $v, u$ the normalisation of $\nu$ restricted to $\Omega^{v, u}$ is a Gibbs state for $F_{v}$ on $\left(\Omega^{v, u}, T^{t[v]}\right)$ and vice versa. Take $s$ to be the lowest common multiple of the numbers $t[v]$.

Proof of the theorem. It follows immediately from lemmas 9 and 10 that $\nu$ is invariant under some power of $T$. As noted at the beginning, the conjugating maps as constructed in lemma 7 give rise to a finite number of homeomorphisms on $(\Omega, T)$. Finally, it is well-known that an equilibrium state on the shift space $\Sigma_{A}$ corresponds automatically to one on $\Omega$ (cf. [4, Theorem 7.9]).

We cannot expect $\nu$ to be an equilibrium state on $(\Omega, T)$, since that would require it to be $T$-invariant. A look at the spectral decomposition as described in lemma 12 shows that this is in general not true. The measure $\nu$ restricted and normalised to $\Omega^{v, u}$ (if $v\left(\Omega^{v, u}\right)>0$ ) is clearly Gibbs and is invariant under $T^{t[v]}$ but not under $T$ unless $t[v]=1$. But $T$ need not be mixing to have an invariant measure. We have:

Corollary 13. (i) A T-invariant Gibbs measure for a Hölder continuous real-valued function over a Smale space is an equilibrium state;

(ii) if T acts topologically mixing, then, by lemma 10, a Gibbs measure for a Hölder continuous real-valued function over a Smale space is an equilibrium state.

I am indebted to Peter Walters for encouragement, advice and especially for pointing out lemma 5.

\section{REFERENCES}

[1] R. Bowen. Equilibrium States and the Ergodic Theory of Anosov Diffeomorphisms. Springer Lecture Notes in Maths. \# 470, 1975.

[2] D. Capocaccia. A definition of Gibbs states for a compact set with $\mathbb{Z}^{\nu}$-action. Comm. Math. Phys. 48, (1976), 85-88.

[3] A. Manning. Axiom A diffeomorphisms have rational Zeta functions. Bull London Math. Soc. 3 (1971), 215-220.

[4] D. Ruelle. Thermodynamic Formalism. Addison-Wesley, 1978. 